\title{
Experimental Evaluation of a Haptic Interface for Endoscopic Simulation
}

\author{
Evren Samur* Lionel Flaction Hannes Bleuler \\ Laboratory of Robotic Systems, Ecole Polytechnique Fédérale de Lausanne (EPFL), Switzerland
}

\begin{abstract}
The main goal of virtual reality based surgery simulators with haptic feedback is to provide an alternative to traditional training methods on animals, cadavers or real patients. Haptic feedback is a key feature for every surgery simulator for the training of hand-eye coordination. To address the need for higher fidelity and complexity in an endoscopic simulator, we have designed a new haptic interface, instrumented a clinical endoscope and integrated it with a software simulation for colonoscopy. The proposed haptic interface provides high translational force and rotational torque with combined electrical motors and passive brakes. This paper presents the evaluation of the haptic interface. Experimental analyzes are performed for characterization and performance evaluation. A model-based feedforward control is implemented and the results show that the control successfully compensates for the device dynamics and nonlinearities such as Coulomb and viscous friction.
\end{abstract}

Index Terms: B.0 [Hardware]: General-Surgery Simulators; B.8.2 [Hardware]: Performance and Reliability-Performance Analysis and Design Aids; C.4 [Computer Systems Organization ]: Performance of Systems-Performance attributes; I.6.3 [Computing Methodologies]: Simulation and Modeling-Applications

\section{INTRODUCTION}

Colonoscopy is a minimally invasive surgery (MIS) procedure where the colon of the patient is examined with an endoscope. It is a complex technical procedure that requires training and experience to maximize accuracy and safety. Computer-based training of this procedure with virtual reality (VR) visualization and haptic feedback offers flexible and repeatable scenarios. In addition, simulators could offer the possibility to precisely assess training progress and even to define levels of skills. Furthermore, it allows session recording and has therefore distinct advantages over traditional training methods on animals, cadavers or real patients. However, existing simulators still need to be improved regarding the realism of the simulation by offering better visualization rendering, adequate force feedback and realistic endoscope simulations

Recently a few studies $[13,14,15,16,25]$ have focused on simulation, modeling and visualization of colonoscopy with haptic feedback. Some of these studies $[2,7]$ have already lead to products. The main drawbacks of todays simulators for a realistic simulation are: absence or a weak force feedback, impossibility to remove the colonoscope from the simulator, coupling of the translational and rotational force feedbacks and bulky designs.

To address the need for higher fidelity and complexity in a colonoscopy simulator, we have designed a new haptic interface [20] and instrumented a clinical colonoscope [17] in order to integrate it with the software simulation framework for colonoscopy (MILX $^{\mathrm{TM}}$ GastroSim) developed at CSIRO $[12,5]$. Several prototypes have been built, presented at medical conferences and commercialization is imminent. This paper presents the evaluation of

*e-mail: evren.samur@epfl.ch

IEEE World Haptics Conference 2011

21-24 June, Istanbul, Turkey

978-1-4577-0298-3/11/\$26.00 @2011 IEEE

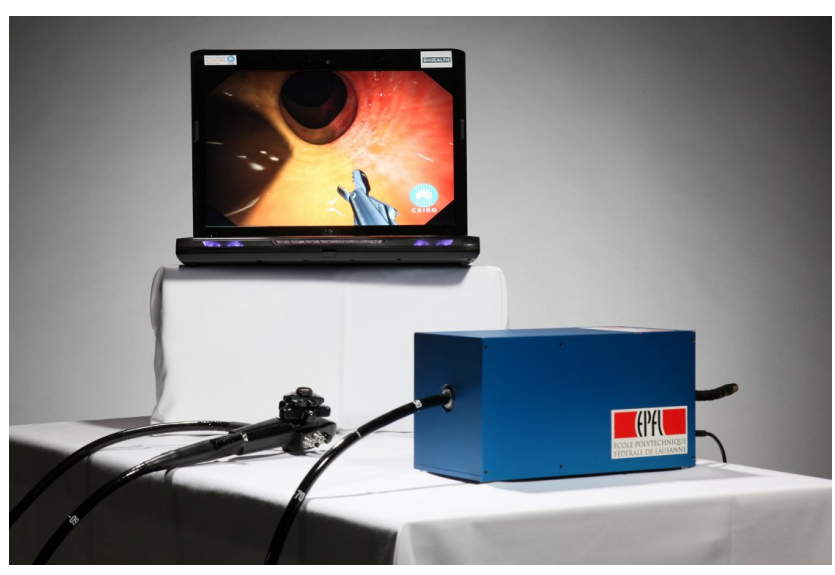

Figure 1: The colonoscopy simulator consists of a 2-DOF haptic interface, an instrumented clinical endoscope and a virtual reality environment to provide real-time visualization.

the haptic interface hardware developed at EPFL (see Figure 1).

The discussion about experimental performance evaluation for haptic interfaces goes back to 80s when force-reflecting hand controllers (today's haptic interfaces) were used in teleoperation. The design requirements for teleoperation were described by Brooks [3] and used by many researchers. Hayward and Astley [11] theoretically defined performance measures directed towards isotonic (i.e. impedance type) devices. More or less at the same time, these measures were formalized for coupled micro-macro actuators by Morrell and Salisbury [19]. In addition, practical ways to measure them were experimentally demonstrated on a haptic interface by Ellis et al. [6]. Several projects $[8,24,26,10]$ evaluated particular haptic devices based on these technical performance metrics. An experimental identification method was described by Frisoli and Bergamasco [9]. Similarly, the dynamics of PHANToM Premium 1.5A (SensAble Technologies Inc.) were experimentally identified by $[4,21]$. Ueberle [23] conducted hardware experiments for the comparative performance evaluation of haptic control schemes using the VISHARD interface [22]. Although a set of performance metrics for haptic interfaces has been defined in literature, there is no consensus on measurement methods which vary considerably between studies. In this study, static and frequency measurements have been chosen to evaluate the haptic interface as they reveal basic characteristics related to actuation and sensing. The experimental methods are described in detail to allow other studies to reutilize the same methods.

\section{HAPTIC INTERFACE}

The developed haptic interface for colonoscopy simulation tracks the position and orientation of a colonoscope and provides force feedback in both translational and rotational directions. Fig. 2 shows a prototype of the haptic interface. The complete electronics for actuators and sensors is integrated on a single card, including the drivers for the actuation. The card is mounted on top of the device, so that only two cables (i.e., a $15 \mathrm{~V}$ power supply and a 


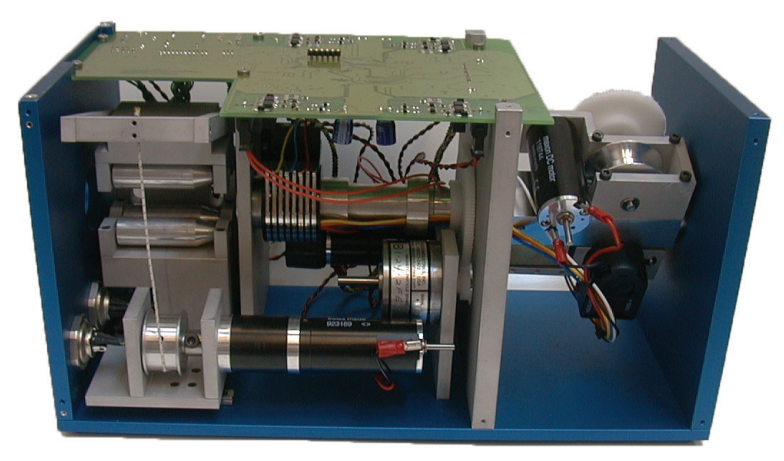

Figure 2: Haptic interface with embedded electronics provides compact and portable solution.

USB cable to the PC) are sufficient to command the device. The $\mathrm{PC}$ runs the virtual reality simulations in parallel to the high-level force control. The communication between the PC and the device is at $1 \mathrm{kHz}$. The force control loop implemented on the card runs at $5 \mathrm{kHz}$ in addition to a $20 \mathrm{kHz}$ PWM loop.

The haptic device includes DC motors for active force feedback and friction compensation as well as brakes for high force rendering. The friction rollers are used to track the axial displacement of the colonoscope and to impose force feedback. One of the friction rollers is connected to a DC motor. The other roller is engaged with an encoder for the tracking of the translational displacement. Decoupling of actuation and tracking systems avoids loss of position tracking data due to an unlikely slippage of the endoscope over the friction rollers. The rotational part is linked to a DC motor by means of gears. RE25 DC motors (Maxon Motor, Switzerland) with a maximum output torque $28.8 \mathrm{mNm}$ are used for active force feedback. An S90MPA-B15D19S magnetic particle brake (Sterling Instrument, USA), which has a maximum torque of $113 \mathrm{mNm}$, is chosen to apply resisting torques on the colonoscope. A custom mechanical brake is designed to be used if forces applied on the colonoscope are too strong to be maintained by the translational motor. The brake system is mounted on linear guidance rails with a displacement of $\pm 5 \mathrm{~mm}$. An attempt to withdraw of the endoscope when the brake is activated results in the release of the brake. Optical encoders are used to track linear and rotational displacements. Considering the transmission ratios, the sensing resolutions of the device are $0.045 \mathrm{~mm}$ and $0.045^{\circ}$ for the two mentioned DOFs.

\section{Experimental Evaluation}

\subsection{Setup}

A $120 \mathrm{~N}$ and $2.0 \mathrm{Nm}$ capacity 6-DOF force/torque sensor (type: Mini40, calibration: SI-40-2, ATI Industrial Automation, Inc.) was used to measure output torque and force. In order to be able to attach the force sensor, the colonoscope was replaced by a $13 \mathrm{~mm}$ diameter and $350 \mathrm{~g}$ metallic rod (the insertion tube of the colonoscope has a weight of 350 gram for the length of $1 \mathrm{~m}$ which is coupled to the device dynamics during normal operation). The surface of the rod was also covered by a plastic heat shrink to obtain similar surface characteristics with the colonoscope. A $\pm 3 g$ triple axis accelerometer (Type: ADXL335, SparkFun Electronics) were attached to the tip of the tube for velocity measurements. Data acquisition is performed at $1 \mathrm{kHz}$ using two NI PCI-cards (one is dedicated to the force sensor). The internal loop at the embedded control card of the haptic interface was set to $5 \mathrm{kHz}$ to be able to generate high frequency excitations.

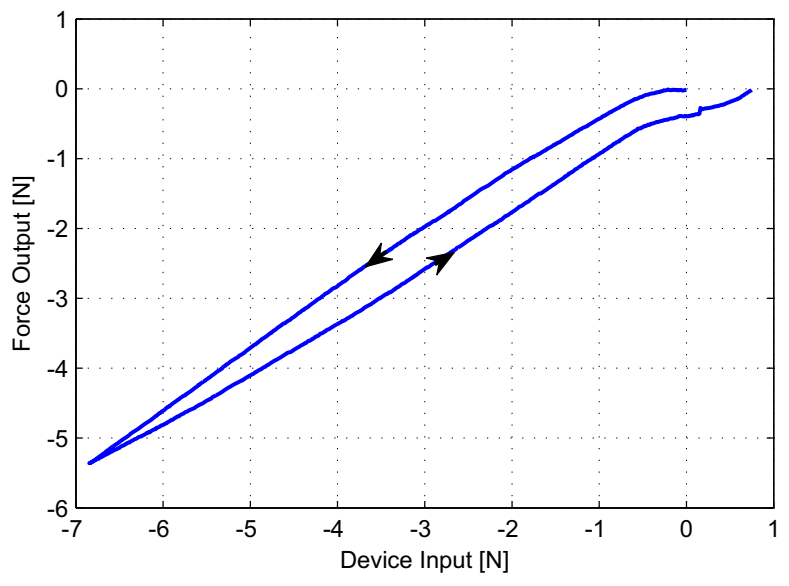

Figure 3: Input-output curve of the translational DOF. The maximum continuous force produced by the DC motor is $5.4 \mathrm{~N}$. The hysteresis loop shows nonlinearity in the device.

\subsection{Static Response Measurements}

One of the most common performance specifications provided by manufacturers is the maximum output capability of a device in static conditions. In the case of haptic interfaces, the most relevant is of course maximum output force. There are two general ways to specify this: transient peak force and maximum continuous force. Since the first one is limited in time due to heat dissipation and actually short in its duration, the maximum continuous force is a better benchmark metric for haptic devices.

In order to measure the continuous output force at the end effector, a slowly increasing and decreasing ramp input was commanded to the motors. Thus, input-output force graphs (i.e., calibration curves) were obtained. As an example, the resulting calibration curve for the translational DOF is shown in Fig. 3. The calibration measurements showed that the maximum continuous force and torque are $5.4 \mathrm{~N}$ and $85 \mathrm{mNm}$, respectively. These maximum values were obtained for the active force feedback. In other words, they are measured when the brakes are not activated. When the brakes are on, the maximum resistive force and torque go up to $80 \mathrm{~N}$ and $300 \mathrm{mNm}$, respectively.

The hysteresis loop in Fig. 3 shows nonlinearity in the actuation system. The possible reasons of this nonlinearity are, first, the Coulomb friction introduced by the friction drive and second, backlash in the gear reduction. The limit of the dead zone near zero gives the minimum force that can be generated by the haptic device which is $0.5 \mathrm{~N}$ in translation and $8 \mathrm{mNm}$ in rotation. These values should not be confused with device resolution. Resolution of an actuation system is the smallest change in an input signal that can be accurately reproduced at the output in the linear operating range. So, the output force resolution of a haptic interface is the smallest incremental force that can be generated in addition to the minimum force. Experimental results showed that the output force resolution of the device is $9 \mathrm{mN}$ for the translation and $0.12 \mathrm{mNm}$ for the rotation.

The calibration curve also gives us a range of operation with a required linearity and accuracy. The dynamic range, which is the ratio of the maximum force to the minimum given in decibels $(\mathrm{dB})$, is calculated as $20 \mathrm{~dB}$ for active force feedback. For passive force feedback (i.e., when the brakes are switched on), this value is as high as $40 \mathrm{~dB}$. 


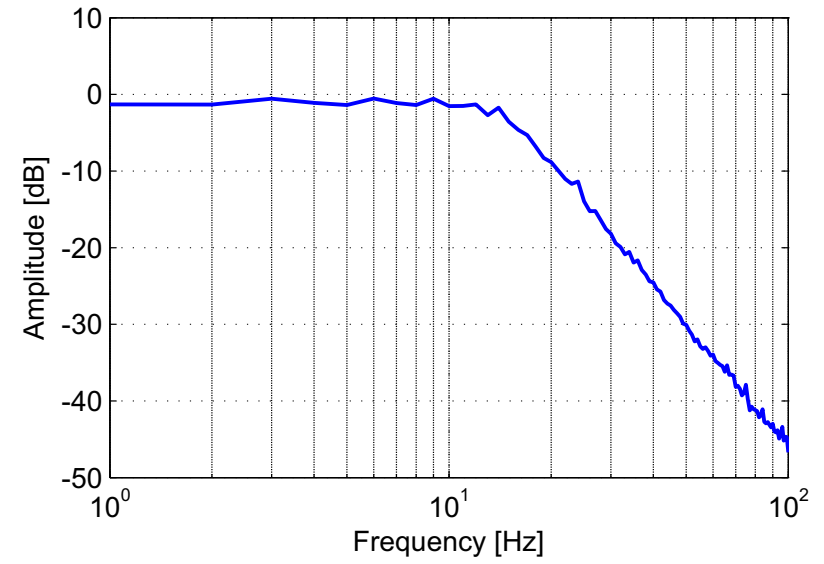

Figure 4: Bode magnitude diagram of $H_{f}$ for the translation DOF. A sweep force is commanded to the motors and the output force is measured when the end effector is constrained.

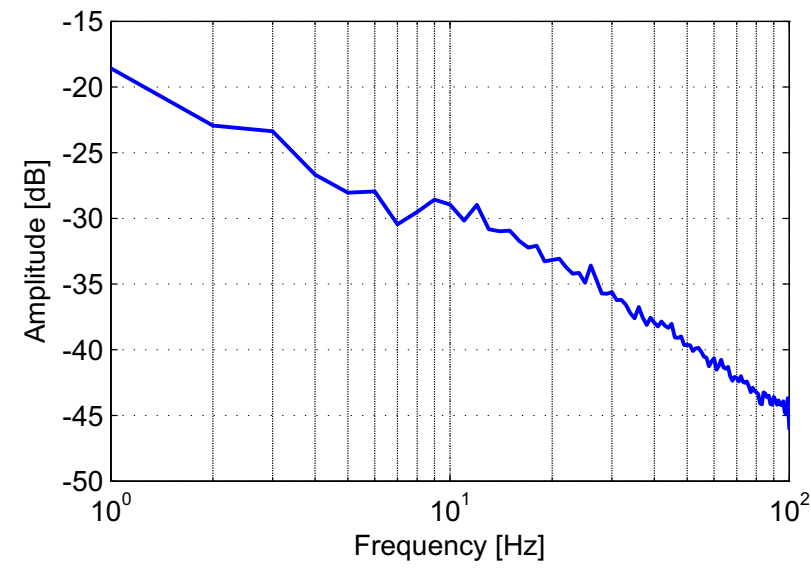

Figure 5: Bode magnitude diagram of $Y_{f}$ for the translational DOF. A sweep force is commanded to the motors and the output velocity is measured when the end effector is free to move.

\subsection{Frequency Response Measurements}

The frequency response of the system was measured by commanding a sweep input (frequency range from $1 \mathrm{~Hz}$ to $100 \mathrm{~Hz}$, amplitude corresponds to maximum force), first, under fixed-end condition (when the end effector is firmly clamped to a rigid, stationary constraint) and then under open-end condition (the detached end effector is free to move). The first one resulted in the transfer function from input force $\left(F_{d}^{n}\right)$ to the output force at the end effector $\left(F_{e e}\right)$ :

$$
\frac{F_{e e}}{F_{d}^{n}}=H_{f}
$$

The Bode diagram corresponding to the transfer function for translation is shown in Fig. 4. The useful frequency range is up to $10 \mathrm{~Hz}$ and the force bandwidth is around $13 \mathrm{~Hz}$.

For the open-ended experiment, accelerometer output was measured while a sweep input was given to the translational motor. Then, the velocity of the end effector was calculated from this signal. The transfer function from the input force to the velocity at the end effector $\left(v_{e e}\right)$ results in the modified admittance of the device:

$$
\frac{v_{e e}}{F_{d}^{n}}=-\frac{H_{f}}{Z_{d}}=-Y_{f}
$$

where $Z_{d}$ is the impedance of the device. The obtained Bode diagram is shown in Fig. 5.

\section{Friction Compensation}

The output force at the end effector is the sum of the desired force and the impedance due the device dynamics for an uncompensated system. Therefore, a feedforward control should be implemented in order to compensate for the device dynamics and to obtain a transparent system as shown below:

$$
F_{e e}=\left(Z_{d}-H_{f} Z_{d}^{n} H_{v}\right) v_{e e}+H_{f} F_{d}^{n}
$$

Here $Z_{d}^{n}$ is an estimation of the device impedance and $H_{v}$ is the transfer function for the velocity measurement.

In order to overcome the impedance (mainly because of the friction in the system), a feed-forward compensation algorithm has been implemented based on a viscous friction model with stiction [18]. Since the velocity resolution of the device was low, a filter with a cutoff frequency of $50 \mathrm{~Hz}$ has been implemented for velocity estimation. This gives a velocity resolution of $2.25 \mathrm{~mm} / \mathrm{s}$.

The performance of the controller was measured while external periodic movements were induced to the tool by the user. The resisting force for the uncontrolled system and for the system under feed-forward control is shown with respect to velocity in Fig. 6. Comparing these results, we can conclude that the friction forces are well compensated with the model-based control. The remaining resistive forces are in the order of $1 \mathrm{~N}$. However, the performance decreases in the low-velocity phase since the control rule highly depends on the velocity calculation. Due to stiction and low velocity resolution of the device, friction forces are higher around zero velocity. To over come this, a zero-crossing algorithm (i.e., a dithering signal to facilitate the stiction breakaway) was developed. However, the vibrations due to this signal were perceivable and disturbing the user. Instead of the dithering, a direction dependent bias force is applied.

\section{Conclusion}

In this paper, a complete approach of evaluating a haptic interface through static and frequency measurements were discussed in detail. These methods were applied to a haptic interface for advanced simulation of colonoscopy. Table 1 summarizes the results of the experimental evaluation study and characteristics of the haptic interface. As seen in the table, when the brakes are turned on (passive
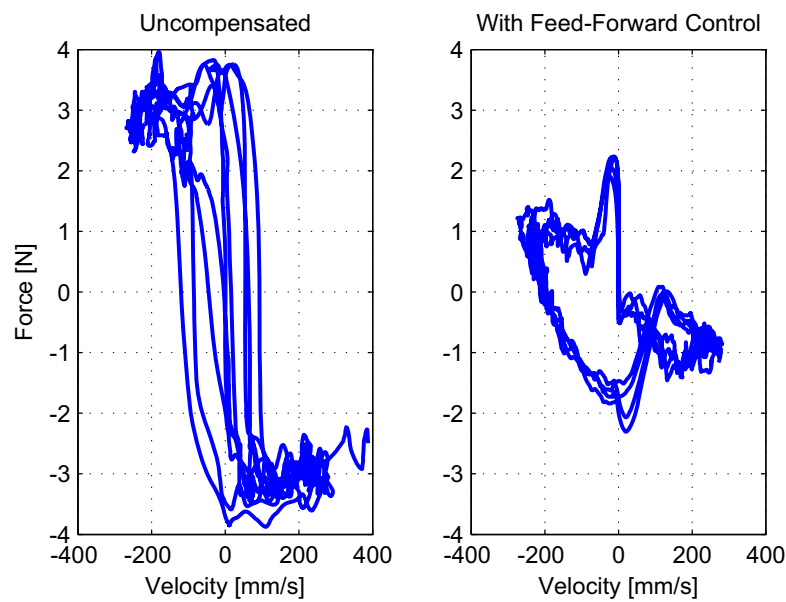

Figure 6: Resisting force in the translation DOF when the user imposes periodic motion to the device: (Left) Without friction compensation, (Right) With feed-forward control. 
Table 1: Characteristics of the Haptic Interface for Colonoscopy Simulation

\begin{tabular}{llrr}
\hline \multirow{2}{*}{ Kinematics } & Metric & Translation & Rotation \\
& DOF & \multicolumn{2}{c}{2} \\
& Structure & \multicolumn{2}{c}{ Hybrid } \\
& Reachable Workspace & $>50 \mathrm{~mm}$ & unlimited \\
\hline \multirow{2}{*}{ Actuation } & Max force (active, passive) & $5.4,80 \mathrm{~N}$ & $85,300 \mathrm{mNm}$ \\
& Min force & $0.5 \mathrm{~N}$ & $8 \mathrm{mNm}$ \\
& Output force resolution & $9 \mathrm{mN}$ & $0.12 \mathrm{mNm}$ \\
& Dynamic range (active, passive) & $20,40 \mathrm{~dB}$ & $20,40 \mathrm{~dB}$ \\
& Force bandwidth & $13 \mathrm{~Hz}$ & $13 \mathrm{~Hz}$ \\
& Useful freq range & $10 \mathrm{~Hz}$ & $10 \mathrm{~Hz}$ \\
\hline \multirow{2}{*}{ Sensing } & Position resolution & $0.045 \mathrm{~mm}$ & $0.045^{\circ}$ \\
& Dynamic range & $\infty$ & $\infty$ \\
\hline
\end{tabular}

feedback), the maximum force and thus dynamic range increase considerably. The force bandwidth of the device is around $13 \mathrm{~Hz}$ which is sufficient to render forces during a typical colonoscopy procedure. These results satisfy the design requirements summarized in [20] except the maximum torque requirement which is 1 $\mathrm{Nm}$ for a procedure performed with an adult size colonoscope [1] Limitation of $0.3 \mathrm{Nm}$ maximum torque in the rotational DOF is due to the slip between the endoscope and the friction rollers. As colonoscopes are designed to slide easily inside the colon, the surface of the colonoscope is very slippery. This issue might be solved by covering either the roller or the endoscope by a suitable material which has higher coefficient of friction. The detailed study of the haptic interface hardware has also shown that the device is subject to nonlinearities such as Coulomb and viscous friction. To overcome this, a feed-forward control has been implemented and the result showed that the control successfully compensates for the device dynamics.

The physical evaluation of the haptic interface provided many useful information about the design and showed the points that should be improved. However, an application-specific evaluation (i.e., clinical trials) should be conducted in order to evaluate how well the device supports the training of colonoscopy procedure. For this purpose, several prototypes have been built and presented at medical conferences. Currently the clinical trials are in progress and the initial results are promising. In addition, commercialization of the whole simulator is imminent.

\section{ACKNOWLEDGEMENTS}

This research has been in part supported by the NCCR Co-Me (Computer Aided and Image Guided Medical Interventions) of the Swiss National Science Foundation.

\section{References}

[1] M. N. Appleyard, C. A. Mosse, T. N. Mills, G. D. Bell, F. D. Castillo, and C. P. Swain. The measurement of forces exerted during colonoscopy. Gastrointest. Endosc., 52(2):237-240, 2000.

[2] S. Bar-Meir. A new endoscopic simulator. Endoscopy, 32(11):898900, 2000.

[3] T. Brooks. Telerobotic response requirements. In IEEE International Conference on Systems, Man and Cybernetics, pages 113-120, Nov 1990.

[4] M. C. Cavusoglu, D. Feygin, and F. Tendick. A critical study of the mechanical and electrical properties of the phantom haptic interface and improvements for high-performance control. Presence: Teleoper. Virtual Environ., 11(6):555-568, 2002.

[5] H. de Visser, J. Passenger, D. Conlan, C. Russ, D. Hellier, M. Cheng, O. Acosta, S. Ourselin, and O. Salvado. Developing a next generation colonoscopy simulator. Int J Image Graphics, 10(2):203-17, 2010.
[6] R. Ellis, O. Ismaeil, and M. Lipsett. Design and evaluation of a highperformance haptic interface. Robotica, 14:321-327, 1996.

[7] EndoscopyVR. CAE Healthcare, 2011. http://www.cae.com/.

[8] E. L. Faulring, J. E. Colgate, and M. A. Peshkin. The cobotic hand controller: Design, control and performance of a novel haptic display. The International Journal of Robotics Research, 25:1099-1119, 2006.

[9] A. Frisoli and M. Bergamasco. Experimental identification and evaluation of performance of a 2 dof haptic display. In Proc. of IEEE International Conference on Robotics and Automation, volume 3, pages 3260-3265, 2003.

[10] R. Gassert, R. Moser, E. Burdet, and H. Bleuler. MRI/fMRIcompatible robotic system with force feedback for interaction with human motion. IEEE/ASME Transactions on Mechatronics, 11(2):216224, April 2006

[11] V. Hayward and O. Astley. Performance measures for haptic interfaces. Robotics Research: The 7th International Symposium, pages 195-207, 1996.

[12] D. Hellier, E. Samur, J. Passenger, U. Spaelter, H. Frimmel, M. Appleyard, H. Bleuler, and S. Ourselin. A modular simulation framework for colonoscopy using a new haptic device. In Proc. of the 16th Medicine Meets Virtual Reality Conference (MMVR), In Press, 2008.

[13] K. Ikuta, K. Iritani, J. Fukuyama, and M. Takeichi. Portable virtual endoscope system with force and visual display. In Proc. of the IEEE/RSJ International Conference on Intelligent Robots and Systems (IROS), volume 1, pages 720-726, 2000.

[14] D. Ilic, T. Moix, N. M. Cullough, L. Duratti, I. Vecerina, and H. Bleuler. Real-time haptic interface for VR colonoscopy simulation. In Proc. of the 13th Medicine Meets Virtual Reality Conference (MMVR), volume 111, pages 208-212, 2005.

[15] O. Korner and R. Manner. Implementation of a haptic interface for a virtual reality simulator for flexible endoscopy. In Proc. of the 11th Symposium on Haptic Interfaces for Virtual Environment and Teleoperator Systems, pages 278-284, 2003.

[16] O. Korner, K. Rieger, and R. Manner. Haptic display for all degrees of freedom of a simulator for flexible endoscopy. Medical Simulation, 3078/2004:161-167, 2004.

[17] P. Maillard, L. Flaction, E. Samur, D. Hellier, J. Passenger, and $\mathrm{H}$. Bleuler. Instrumentation of a clinical colonoscope for surgical simulation. In Engineering in Medicine and Biology Society, 2008. EMBS 2008. 30th Annual International Conference of the IEEE, pages 7073, Aug. 2008.

[18] T. Moix. Mechatronic elements and haptic rendering for computerassisted minimally invasive surgery training. PhD Thesis EPFL, No. 3306, 2005.

[19] J. B. Morrell and J. K. Salisbury. Parallel-coupled micro-macro actuators. The International Journal of Robotics Research, 17:773-791, 1998.

[20] E. Samur, F. Lionel, U. Spaelter, H. Bleuler, D. Hellier, and S. Ourselin. A haptic interface with motor/brake system for colonoscopy simulation. In Proc. of the Symposium on Haptic Interfaces for Virtual Environment and Teleoperator Systems, pages 477- 
478, 2008.

[21] B. Taati, A. M. Tahmasebi, and K. Hashtrudi-Zaad. Experimental Identification and Analysis of the Dynamics of a PHANToM Premium 1.5A Haptic Device. Presence: Teleoperators \& Virtual Environments, 17(4):327-343, 2008.

[22] M. Ueberle, N. Mock, and M. Buss. Vishard10, a novel hyperredundant haptic interface. In Haptic Interfaces for Virtual Environment and Teleoperator Systems, 2004. HAPTICS '04. Proceedings. 12th International Symposium on, pages 58-65, March 2004.

[23] M. W. Ueberle. Design, control, and evaluation of a family of kinesthetic haptic interfaces. PhD Thesis, Technische Universitt Mnchen, 2006.

[24] J. F. Veneman, R. Ekkelenkamp, R. Kruidhof, F. C. van der Helm, and H. van der Kooij. A series elastic- and bowden-cable-based actuation system for use as torque actuator in exoskeleton-type robots. The International Journal of Robotics Research, 25:261-281, 2006.

[25] S. Y. Yi, H. S. Woo, W. J. Ahn, J. Y. Kwon, and D. Y. Lee. New colonoscopy simulator with improved haptic fidelity. Journal Advanced Robotics, 20(3):349-365, March 2006.

[26] J. Yoon and J. Ryu. Design, fabrication, and evaluation of a new haptic device using a parallel mechanism. IEEE/ASME Transactions on Mechatronics, 6(3):221-233, 2001. 